\title{
Helicity of Magnetic Clouds and Their Associated Active Regions
}

\author{
Robert J. Leamon and Richard C. Canfield \\ Department of Physics, Montana State University, Bozeman, MT \\ 59717-3840 USA.
}

Sarah L. Jones

Department of Physics and Astronomy, Dartmouth College, Hanover, NH 03755 USA.

Keith Lambkin

Experimental Physics Department, University College Dublin, Dublin 4, Republic of Ireland.

Brian J. Lundberg

Department of Physics, The University of Arizona, Tucson, AZ 85721 USA

\author{
Alexei A. Pevtsov \\ National Solar Observatory, Sunspot, NM 88349 USA.
}

\begin{abstract}
Magnetic clouds are associated with many Coronal Mass Ejections. Many CMEs involve active regions. In this work we focus on the relationship between twelve magnetic clouds and their associated active regions. We use a cylindrically symmetric constant- $\alpha$ force-free model to derive field line twist, total current, and total magnetic flux from in situ observations of magnetic clouds. We compare these properties with those of the associated solar active regions, which we infer from solar vector magnetograms.

Our comparison of fluxes and currents reveals: (1) the total (unsigned) flux ratios $\Phi_{M C} / \Phi_{A R}$ tend to be of order unity; (2) the total flux ratios tend to be orders of magnitude larger than the total (unsigned) current ratios $I_{M C} / I_{A R}$; and (3) there is a statistically significant proportionality between them. Our key findings in comparing total twists $\alpha L$, where $L$ is the axial dimension of the system, are: (1) the values of $(\alpha L)_{M C}$ are typically an order of magnitude greater than those of $(\alpha L)_{A R}$; and (2) there is no systematic sign or amplitude relationship between them. These findings compel us to believe that magnetic clouds associated with active region eruptions are formed by magnetic reconnection between these regions and their larger-scale surroundings, rather than pre-existing structures in the corona or chromosphere.
\end{abstract}

